\title{
Estudio e intervención en niños con Trastorno por Déficit de Atención e Hiperactividad de educación primaria
}

\author{
Datos de contacto: \\ Leticia Fernández Gacho \\ Doctorando \\ leti_myrg@hotmail.com \\ Víctor Arias González \\ PTUN \\ Victor.arias@gmail.com \\ Henar Rodríguez Navarro \\ PTUN \\ henarrod@pdg.uva.es \\ Nuria Manzano Soto \\ PTUN \\ nmanzano@uned.es
}

\author{
Leticia FERNÁNDEZ GACHO \\ Víctor ARIAS GONZÁLEZ \\ Henar RODRÍGUEZ NAVARRO \\ Nuria MANZANO SOTO
}

\section{RESUMEN}

El presente trabajo aborda el estudio de dos niños con TDAH (Trastorno por Déficit de Atención con Hiperactividad) de 10 y 11 años, en el ámbito escolar. Revisiones previas como Lora y Moreno (2008); Ronk, Hund y Landau (2011); Bishop, Mulraney, Rinehart y Sciberras (2019); Willis, Siceloff, Morse, Neger y Flory (2019) o Fox, Dishman, Valicek, Ratcliff y Hilton (2020), reflejaron la necesidad de llevar a cabo nuevos programas y enfoques de intervención social, para mejorar la capacidad social de estos escolares. La aportación de este estudio se centra en un programa de intervención de las habilidades sociales en escolares con TDAH desde un enfoque conductual. En este artículo presentamos una intervención psicosocial donde aparecen ciertos indicadores que consideramos relevantes para el tratamiento adaptativo de dicho trastorno (habilidades sociales: integración, resolución de conflictos, comunicación e interacción social, y comportamiento: conducta disruptiva, rabietas, frustración, autocontrol y normas). La intervención, puesta en práctica por la investigadora en colaboración con la tutora, consta de 15 sesiones de dos horas semanales, durante 4 meses. Utilizamos un estudio de casos, de corte instrumental (Stake, 1998) y se realiza un análisis de datos anidado (Creswell, 2014), donde el estudio cualitativo guía al cuantitativo. Los resultados apuntan hacia la mejora en el ámbito social del alumnado con TDAH y una necesidad de intervenir también en las acciones concretas para modificar la conducta. Se pretende que este programa pueda servir como orientación para futuras investigaciones, que necesiten aplicar programas de intervención con TDAH en muestras más amplias.

PALABRAS CLAVE: TDAH, educación primaria, intervención, habilidades sociales y conducta. 


\title{
Study and intervention in children with Attention Deficit Hyperactivity Disorder in primary education
}

\begin{abstract}
This papel addresses the study of two children with ADHD (Attention Deficit Hyperactivity Disorder) aged 10 and 11 at school. Previous reviews such as Lora and Moreno (2008); Ronk, Hund and Landau (2011); Bishop, Mulraney, Rinehart and Sciberras (2019); Willis, Siceloff, Morse, Neger and Flory (2019) or Fox, Dishman, Valicek, Ratcliff and Hilton (2020), reflected the need for new social intervention programmes and approaches, to improve the social capacity of these school children. The contribution of this study focuses on a program of intervention of social skills in school children with ADHD from a behavioral approach.

In this article we present a psychosocial intervention where there are certain indicators that we consider relevant to the treatment of said disorder (social skills: integration, conflict resolution, communication and social interaction, and behavior: disruptive behavior, tantrums, frustration, self-control and compliance). The intervention, implemented by the researcher in collaboration with the tutor, consists of 15 sessions of two hours per week, for 4 months. We use a case study, instrumental cut (Stake, 1998) and a nested data analysis is performed (Creswell, 2014), in this case the qualitative study guides the quantitative.

Results point to improvement in the social field of ADHD students and a need to intervene in concrete actions to change behavior as well. It is intented that this program can serve as guidance for future research, who need to apply ADHD intervention programs in larger samples.
\end{abstract}

KEYWORDS: ADHD, primary education, intervention, social skills and behavior.

\section{Introducción}

El Trastorno por Déficit de Atención e Hiperactividad (TDAH) es uno de los trastornos más comunes en la infancia y con mayor presencia en los centros escolares.

Los primeros datos oficiales datan de 1775 cuando Melchor Adam Weikard describió el trastorno de la atención, considerando a estas personas como fácilmente distraibles e hiperactivas. Sin embargo, fue Alexander Crichton (1798), quién describió las características de lo que se entiende hoy en día por TDAH inatento (Vinuesa-Fernández, 2017).

Aun así, la primera descripción científica del trastorno la hizo George Frederic Still en 1902, cuando estudió a niños con actividades impulsivas excesivas y sin alteraciones asociadas (Parellada, 2009). Still lo consideraba como un "defecto patológico en el control moral", cuya 
atención estaba supeditada a estímulos que les ofrecian una gratificación inmediata. Podríamos considerarlo como el primero en hablar de este trastorno, pues describia los síntomas de lo que hoy se considera TDAH de tipo combinado (Lakoff, 2000; citado por LondoñoParedes, 2017).

En la actualidad, se considera como:

Un trastorno de inicio en la infancia que comprende un patrón persistente de conductas de desatención, hiperactividad e impulsividad. Se considera que el trastorno está presente cuando estas conductas tienen mayor frecuencia e intensidad de lo que es habitual según la edad y el desarrollo de la persona, y tales manifestaciones interfieren de forma significativa en el rendimiento escolar o laboral, y en sus actividades cotidianas. (APA, 2013, p.99).

Del mismo modo, a lo largo de esa historia son diversas las causas atribuidas al TDAH; considerándose hoy en dia un trastorno multifactorial, donde se reflejan factores genéticos, biológicos, psicosociales, neuroquímicos y neuroanatómicos.

Debido a esta larga evolución y a su aparición tan temprana, el TDAH es uno de los trastornos con mayor prevalencia en la población infantil con un 5\% según el DSM-5 (APA, 2013) y un 8\% según CIE-10 (OMS, 1992).

Tradicionalmente sus síntomas principales son el déficit de atención, hiperactividad e impulsividad. Además de estos sintomas hay que tener en cuenta los niveles conductuales y cognitivos (Garrido-Landivar, 2017). Desde ahí se ha enfocado el trabajo, desde el aspecto conductual y las habilidades sociales, pues muchos de estos sujetos destacan por sus malas relaciones, como asi lo reflejan investigaciones previas como la de Liesa-Orús, Latorre-Cosculluela y Vázquez-Toledo (2017); Aduen, Day, Kofler, Harmon, Wells y Sarver (2018); Kofler, Harmon, Aduen, Day, Austin, Spiegel, Irwin y Sarver (2018) o Fox, Dishman, Valicek, Ratcliff y Hilton (2020). Sus problemas de conducta hacen que tengan problemas de integración social, como levantarse constantemente en medio de una explicación, no parar de moverse en su sitio molestando a sus compañeros o actuar con impulsividad. Es importante abordar estos aspectos, sino seguirán siendo vistos como molestos, afectando a su sociabilidad.

Debido a esta variedad sintomatológica, el diagnóstico del TDAH es multifactorial, abarcando aspectos como entrevistas, exámenes médicos, historia médica y familiar, escalas o test; el diagnóstico requiere la recopilación de toda la información posible sobre la conducta del niño, ya que no existen marcadores biológicos. En este estudio se han utilizado las entrevistas, observación, cuestionarios, así como información del equipo de orientación sobre la historia clínica y educativa.

En cuanto a los tratamientos, existe una gran variedad derivados de enfoques distintos: el farmacológico, el cognitivo-conductual y el 
combinado. Este estudio se centra en el cognitivo-conductual, pues como refleja el estudio de Storebo, Elmose-Andersen, Skoog, JoostHansen, Simonsen, Pedersen, Tendal, Callesen, Faltinsen y Gluud, (2019) el farmacológico puede aliviar los síntomas del TDAH, pero rara vez resuelve los problemas sociales, por eso es importante destacar el cognitivo-conductual.

Esos problemas sociales en niños con TDAH, se han destacado a lo largo de los años por varios estudios como Lora y Moreno (2008), Ronk, Hund y Landau (2011) y Puentes-Rozo, Jiménez-Figueroa, Pineda-Alhucema y Montoya (2014); o estudios más recientes como el de Aduen, Day, Kofler, Harmon, Wells y Sarver (2018) o Fox, Dishman, Valicek, Ratcliff y Hilton (2020). A partir de ahí se ha enfocado este trabajo, para abordar las dificultades sociales que estos niños pudieran tener. Estos niños suelen tener dificultades interpersonales y baja competencia social. Sus sintomas principales interfieren en su ámbito social, pues molestan, hablan, son quietos... provocando el alejamiento o el rechazo de las personas que les rodean.

Además, el déficit en las funciones ejecutivas que presentan los sujetos con TDAH hace que tengan peor inatención, autocontrol $y$, en consecuencia, malas habilidades sociales (Becker, Garner, Tamm, Antonini y Epstein, 2019).

El modelo híbrido de Barkley (1997) refleja cómo estos sujetos dedican poco tiempo a sus interacciones sociales, conllevando a deficiencias en sus habilidades sociales y en sus conductas adaptativas (Puentes-Rozo, et al., 2014). Storebo, Elmose-Andersen, Skoog, Joost-Hansen, Simonsen, Pedersen, Tendal, Callesen, Faltinsen y Gluud (2019) reflejaron la necesidad de llevar a cabo más ensayos sobre el entrenamiento de las habilidades sociales en sujetos con TDAH, de ahí este pequeño paso llevado a cabo con este estudio, para poder ser replicado en futuros estudios con mayores muestras. En esta línea ya destacaban Peña et al. en 2009, quienes concluyeron que la intervención más adecuada es aquella que combina el tratamiento farmacológico con medidas psicosociales, destacando el entrenamiento en habilidades sociales (Rojo-Amato, Tárraga-Mínguez y Sanz-Cervera, 2016).

Resulta de interés la implementación de programas de intervención dirigidos a potenciar las habilidades sociales (Russo, Bakker, Rubiales y Betina-Lacunza, 2019), por ello el objetivo del presente trabajo fue la presentación de los resultados preliminares de un programa de intervención en habilidades sociales y dificultades conductuales en dos casos de TDAH. Además, se tuvo en cuenta el ámbito escolar, pues es donde más horas al día pasan y donde más relaciones establecen.

\section{Objetivo}

Valorar los efectos de una intervención basada en las habilidades sociales y en la conducta en dos casos de TDAH en el ámbito escolar. 
Concretamos dicho objetivo con las preguntas operativas:

¿Se puede conseguir una mejora en las habilidades sociales en estos casos con una intervención escolar?

Y en concreto: ¿Existen evidencias desde la literatura científica para demostrar que los escolares con TDAH tienen afectadas las habilidades sociales?

¿Tienen estos casos con TDAH afectadas las habilidades sociales?

¿Qué efectos tiene esta intervención en los aspectos sociales de estos niños?

¿Es necesario intervenir en ciertos aspectos conductuales relacionados con el TDAH para conseguir beneficios sociales?

¿Se puede llevar a cabo una intervención en el ámbito escolar sin alterar la dinámica académica?

En consonancia con ello, se tuvieron en cuenta investigaciones previas como la de Becker et al., (2019), Willis, Siceloff, Morse, Neger y Flory, (2019) y Fox et al., (2020), entre otras.

\section{Método}

Seleccionamos el estudio de caso intrínseco (Stake, 1998), como estructura metodológica desde donde abordar esta investigación. Además unimos el enfoque de Creswell sobre métodos mixtos para analizar los datos desde una perspectiva anidada (Creswell, 2014).

Se trata de un caso típico (Stake, 1998), dos niñas con TDAH con dificultades sociales y conductuales dentro del ámbito académico. Estos casos tienen aspectos comunes que nos permitieron unificar determinados resultados: dificultades sociales (retraimiento, rechazo, reducida red social) y conductuales (impulsividad conductual, escasa autorregulación), variables que son analizadas en el estudio.

El análisis de datos es un enfoque mixto, anidado, donde se combinan aspectos cuantitativos y cualitativos, para responder a un problema de investigación, relacionando la recogida, análisis e interpretación (Creswell, 2014). Este método nos permite enriquecer la información pues se puede complementar y mejorar su interpretación.

\section{Participantes}

El estudio está centrado en dos alumnas de educación primaria, de edades comprendidas entre los 10 y 11 años. Se elige este nivel educativo porque es donde se empiezan a ver mayores dificultades.

- Uno de los casos está diagnosticado como ACNEE con TDAH de tipo combinado. A los 14 meses se la diagnosticó retraso en el desarrollo motor grueso y con 2 años epilepsia, solo cuando tenía fiebre. En la actualidad solo se observa la epilepsia.

A nivel escolar, refleja capacidad intelectual límite y dificultades sociales. 
No toma medicación para el TDAH, porque toma para la epilepsia.

- El otro caso está diagnosticado como ACNEE con TDAH con predominio de inatención.

A nivel escolar, hubo un cambio de centro porque la hacían Bullying. Desde orientación reflejan una capacidad intelectual límite, así como una conducta y comportamiento inadecuado: rabietas, desconfianza, miedo a lo desconocido, frustración, actos impulsivos de agresión hacia compañeros y profesores, reacciones inesperadas y desproporcionadas.

No toma mediación pues afecta a su estado de ánimo (apatía y tristeza).

\section{Diseño}

A nivel metodológico, seleccionamos un estudio de caso, pues tiene interés en sí mismo y es un estudio de un caso singular, para comprender su actividad en determinadas circunstancias (Stake, 1998). Dado que el objetivo de este estudio es analizar las mejoras de dos casos de TDAH en el contexto escolar tras una intervención, el estudio de caso permite estudiar en profundidad ambos casos, sus situaciones y circunstancias que les rodean, para poder intervenir con posterioridad y valorar las posibles mejoras.

En este caso, el estudio de caso está centrado en los aspectos sociales y conductuales del TDAH y nos permite realizar su estudio de manera exhaustiva, para poder generalizarlo en posteriores ocasiones a una muestra mayor.

El enfoque desarrollado en el análisis de datos es de carácter mixto. En las Ciencias Sociales y aún más, en las Habilidades Sociales, surgen comportamientos y acciones subjetivas que no pueden medirse solo de forma cuantitativa, de ahí la necesidad de realizar un método mixto, anidado. Éste nos permite utilizar ambos métodos, donde uno guía al otro (Creswell, 2014), utilizando datos cuantitativos y cualitativos para responder al problema de investigación. De esta manera la información se combina, complementa y mejora.

Teniendo en cuenta todo lo anterior, si se aplicase solo la metodología cualitativa se nos quedaría corto para evaluar el estudio. Del mismo modo, utilizar solo la metodología cuantitativa no nos permitiría recoger aspectos importantes como el contexto que rodea los casos y cómo se desenvuelven en su ambiente natural, o contar con las opiniones de las personas que las rodean. Al ser un estudio de caso y tener que realizar un análisis exhaustivo de las situaciones, era necesario contar con técnicas cualitativas como la observación y las entrevistas a padres y tutores; y con técnicas cuantitativas que nos permitieran contrastar los datos y proporcionar información adicional, como los cuestionarios y los sociogramas. De esta manera se conseguiría un estudio más completo y permitiría evitar ciertos sesgos de interpretación en el investigador. 


\section{Instrumentos}

A continuación se refleja la tabla 1 con los principales instrumentos utilizados, los objetivos y el tipo de resultado obtenido.

Tabla 1.

Instrumentos utilizados

\begin{tabular}{|c|c|c|c|}
\hline Técnica & Objetivo & Tipo de resultado & $\begin{array}{l}\text { Herramientas } \\
\text { de análisis }\end{array}$ \\
\hline $\begin{array}{l}\text { Observación: } \\
\text { diario de campo }\end{array}$ & $\begin{array}{l}\text { Obtener } \\
\text { información de } \\
\text { sus habilidades } \\
\text { sociales y } \\
\text { conducta en el } \\
\text { ambiente natural }\end{array}$ & $\begin{array}{l}\text { Reflejo fiel de la realidad } \\
\text { Registro de tres } \\
\text { aspectos: afectivos, } \\
\text { conductuales y sociales } \\
\text { Registro de sensaciones } \\
\text { e impresiones }\end{array}$ & Nudist Nvivo 8 \\
\hline Entrevista & $\begin{array}{l}\text { Obtener } \\
\text { información de } \\
\text { padres y tutores }\end{array}$ & $\begin{array}{l}\text { Entrevista } \\
\text { semiestructurada } \\
\text { Captar los aspectos } \\
\text { sociales, conductuales y } \\
\text { de TDAH }\end{array}$ & Nudist Nvivo 8 \\
\hline Sociogramas & $\begin{array}{l}\text { Buscar las } \\
\text { relaciones } \\
\text { sociales en clase }\end{array}$ & $\begin{array}{l}\text { Cohesión grupal } \\
\text { Posición social de los } \\
\text { alumnos dentro del } \\
\text { grupo: líder, rechazado... } \\
\text { Relaciones sociales } \\
\text { existentes: positivas y } \\
\text { negativas }\end{array}$ & SociometryPro \\
\hline $\begin{array}{l}\text { Cuestionarios } \\
\text { de habilidades } \\
\text { sociales: CABS, } \\
\text { Goldstein y } \\
\text { Atención a la } \\
\text { Diversidad }\end{array}$ & $\begin{array}{l}\text { Información de } \\
\text { las habilidades } \\
\text { sociales en } \\
\text { general antes y } \\
\text { después de la } \\
\text { intervención }\end{array}$ & $\begin{array}{l}\text { Conducta socialmente } \\
\text { hábil } \\
\text { Asertividad } \\
\text { Información de los } \\
\text { propios casos de TDAH, } \\
\text { padres y tutores }\end{array}$ & $\begin{array}{l}\text { Propio sistema } \\
\text { de corrección }\end{array}$ \\
\hline
\end{tabular}

Todos estos métodos de recogida de información han aportado información amplia, que permite ser más rigurosos con los datos observados, así como su triangulación.

\section{Procedimiento.}

De esta intervención destacamos como aspectos relevantes para el tratamiento educativo del TDAH: el entrenamiento en las habilidades sociales y la intervención en sus comportamientos disruptivos, impulsivos e inatentos, que provocan problemas sociales.

Se hipotetizó que la mejora de sus habilidades sociales podía traer 
mejoras en los sintomas generales del TDAH, y al revés, si se controlaban los sintomas del TDAH se producirian mejoras en su conducta y en sus habilidades sociales.

Durante la investigación se llevaron a cabo varias fases, que se presentan en la tabla 2 de forma resumida.

Tabla 2 .

Fases del procedimiento llevado a cabo

\begin{tabular}{lll}
\hline Fases & Intervención & Actividades realizadas \\
\hline Fase 1 & Recogida de & Entrevistas a padres y profesores. \\
& información e & Observación en el ambiente natural. \\
& Instrumentos & Sociogramas. \\
& & Cuestionarios de habilidades sociales (sujetos con \\
& & TDAH, padres y tutores).
\end{tabular}

Fase 2 Entrenamiento Explicación del programa.

tutores Explicación del uso de las técnicas sociales y conductuales.

Fase 3 Intervención 4 meses.

2 horas a la semana.

Sesión individual (horas de apoyo) y sesión grupal (tutorias).

Uso de recreos para la cohesión grupal.

Cada semana era una temática

Fase 4 Recogida de Mismo sistema de recogida de datos inicial información

La intervención constaba de 15 sesiones temáticas donde se ahondaron conocimientos relacionados con las habilidades sociales y conducta. A continuación aparecen en la tabla 3.

Tabla 3.

Sesiones incluidas en la intervención

\begin{tabular}{ll}
\hline Sesiones & Temática \\
\hline Sesión 1 & Apertura y acercamiento. \\
Sesión 2 & Derechos y deberes. \\
Sesión 3 & Sociabilidad. \\
Sesión 4 & Asertividad. \\
Sesión 5 & Empatia. \\
Sesión 6 & Emociones y sentimientos. \\
Sesión 7 & Autoestima y autoconcepto. \\
Sesión 8 & Comunicación verbal y no verbal \\
\hline
\end{tabular}




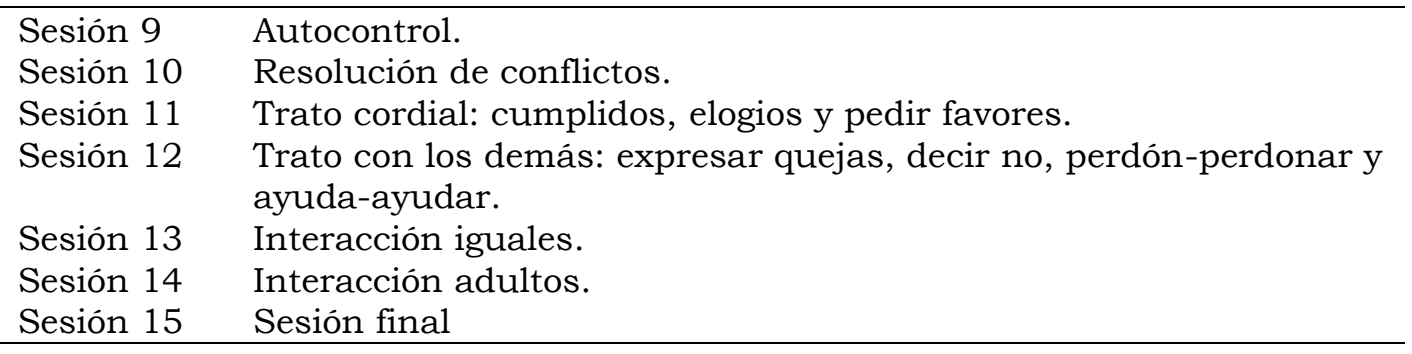

Todas estas sesiones fueron llevadas a cabo por la investigadora. En ellas se explicaba el concepto a trabajar esa semana, se llevaban a cabo dinámicas para ponerlo en práctica y, en las sesiones individuales, se mandaban actividades para casa a los dos casos de TDAH, siempre relacionadas con la temática trabajada (videos, cuentos, prácticas...). Del mismo modo, los profesores aplicaron en el aula actividades relacionadas con la temática semanal para reforzar la intervención a modo de dinámicas, como técnicas de autocontrol (tortuga o respiración), emociones (diario de emociones) entre otras; o de modificación de conducta (extinción, tiempo fuera...). De esta manera, cada semana la clase entera ahondaba en una temática.

Las sesiones se llevaron a cabo en las horas de tutoría y apoyo enlazando con los recreos, para evitar perder el ritmo académico y también por considerarse enriquecedoras para aplicar en esos momentos.

Además, se utilizaron estrategias para controlar determinados síntomas del TDAH, pues se estableció que controlando esos síntomas (levantarse constantemente, molesta e interrumpe, levanta constantemente la mano, juguetea, es incapaz de jugar tranquilamente, se distrae, responde impulsivamente, no espera su turno, inquietud, excitabilidad o habla cuando no debe) se podian controlar estas variables, permitiendo mejorar su conducta y habilidades sociales.

Muchas de estas estrategias están basadas en técnicas de modificación de conducta, como el refuerzo positivo, o técnicas creadas para controlar los sintomas del TDAH, como pactar momentos en los que puede levantarse, para evitar hacerlo cuando no debe.

Con la intervención en estos momentos se puede demostrar la viabilidad de realizar el programa por parte de los tutores en un futuro.

El papel de los padres también fue relevante, pues a pesar de que la intervención estaba centrada en el ámbito académico, la aplicación de técnicas conductuales en casa permitía complementar la intervención.

\section{Resultados}

Los resultados obtenidos permitieron aclarar el objetivo de partida y responder a las preguntas iniciales. Se pudo ver los efectos de una intervención basada en las habilidades sociales y conducta en casos 
de TDAH en el ámbito escolar. Al mismo tiempo se pudo dar respuesta a la pregunta: ¿Se puede conseguir una mejora en las habilidades sociales en estos casos con una intervención escolar?

Los datos obtenidos de las entrevistas y observación reflejaron que en ambos casos se obtuvieron dificultades iniciales en sus capacidades sociales generales.

Los dos casos reflejaron comportamientos que conllevaron al rechazo por parte de sus compañeros. Esto genera dificultades en sus interacciones sociales, como se muestra en la tabla 4, donde aparecen resumidos los principales aspectos de estas alumnas a nivel conductual y referidos al TDAH.

Tabla 4.

Clasificación de las conductas

\begin{tabular}{|c|c|c|}
\hline & $\begin{array}{l}\text { Alumna } 1 \\
\end{array}$ & Alumna 2 \\
\hline Conducta & $\begin{array}{l}\text { Molesta y pega a los demás } \\
\text { Tiene rabietas } \\
\text { Molesta e interrumpe } \\
\text { Problemas de autocontrol } \\
\text { Tiene enfrentamientos }\end{array}$ & $\begin{array}{l}\text { Tiene rabietas } \\
\text { Cambios del estado de ánimo } \\
\text { Frustración } \\
\text { Problemas de autocontrol } \\
\text { Tiene enfrentamientos } \\
\text { Pega a los demás }\end{array}$ \\
\hline $\begin{array}{l}\text { Sintomas } \\
\text { asociados } \\
\text { al TDAH }\end{array}$ & $\begin{array}{l}\text { No piensa las consecuencias de } \\
\text { sus acciones } \\
\text { Juguetea } \\
\text { Se levanta constantemente } \\
\text { Levanta la mano } \\
\text { constantemente } \\
\text { Incapaz de jugar } \\
\text { tranquilamente } \\
\text { Baja persistencia en las tareas } \\
\text { Responde impulsivamente } \\
\text { Sus demandas deben } \\
\text { satisfacerse inmediatamente } \\
\text { Problemas para esperar su } \\
\text { turno } \\
\text { Inquietud } \\
\text { Excitabilidad }\end{array}$ & $\begin{array}{l}\text { No piensa en las consecuencias } \\
\text { de sus acciones } \\
\text { Juguetea constantemente } \\
\text { Incapaz de jugar tranquilamente } \\
\text { Baja persistencia en las tareas } \\
\text { Responde impulsivamente } \\
\text { Inquietud } \\
\text { Excitabilidad } \\
\text { Sus demandas deben satisfacerse } \\
\text { inmediatamente }\end{array}$ \\
\hline
\end{tabular}

¿Qué efectos tiene esta intervención en los aspectos sociales de los niños con TDAH?

Este aspecto se valoró con las pruebas anteriormente comentadas, aplicadas antes y después de la intervención.

Los sociogramas fueron elaborados contando con dos aspectos fundamentales: académicos y sociales. Estos nos permitieron valorar la 
preferencia y no preferencia para Trabajar en Grupo, pasar el Tiempo Libre y Sentarse en Clase.

Los sociogramas se aplicaron a sus respectivos grupos de clase.

Con esta evaluación pretendíamos obtener la situación social de las alumnas dentro de clase. Esto permitía valorar cómo era su integración con el grupo-clase y cómo eran las relaciones sociales en el ámbito académico. Esta información es muy útil para comprobar sus habilidades sociales escolares antes y después de la intervención. Estos datos se van a reflejar en tablas comparativas, en función de las tres variables evaluadas, comentadas con anterioridad.

En lo referente a la alumna 1, el Trabajo en Grupo se refleja en la tabla 5.

Tabla 5.

Resultado sociograma Trabajar en Grupo

\begin{tabular}{cll}
\hline $\begin{array}{c}\text { Elecciones } \\
\text { positivas }\end{array}$ & $\begin{array}{l}\text { Entes el primer sociograma, } \\
\text { Gráfico 1, ningún compañero } \\
\text { la eligió para Trabajar en } \\
\text { Grupo. Hasta 8 alumnos no } \\
\text { se han tenido en cuenta. }\end{array}$ & $\begin{array}{l}\text { Tras la intervención, Gráfico 2,3 } \\
\text { compañeros la han elegido para } \\
\text { trabajar en grupo }\end{array}$ \\
$\begin{array}{c}\text { Elecciones } \\
\text { negativas }\end{array}$ & $\begin{array}{l}\text { En el primer sociograma, } \\
\text { Gráfico 3, 15 compañeros no } \\
\text { la quieren tener en su grupo. }\end{array}$ & $\begin{array}{l}\text { Mientras que en el segundo } \\
\text { sociograma, Gráfico 4, se puede } \\
\text { observar que 4 alumnos han } \\
\text { elegido a Ana de forma negativa. }\end{array}$
\end{tabular}

Estos resultados se reflejan a continuación en forma de gráficos, donde aparecen las elecciones positivas y negativas antes y después de la intervención. 


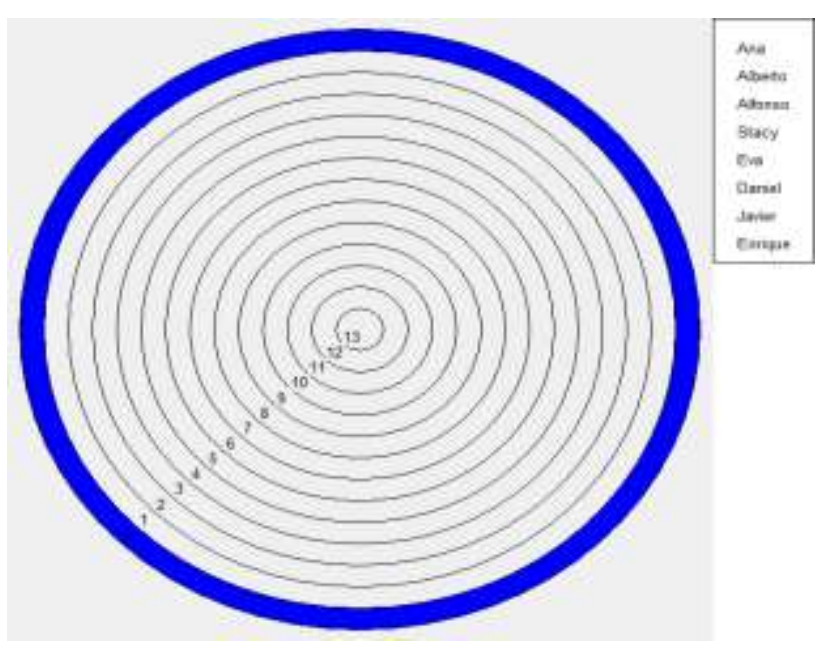

Gráfico 1. Elecciones positivas

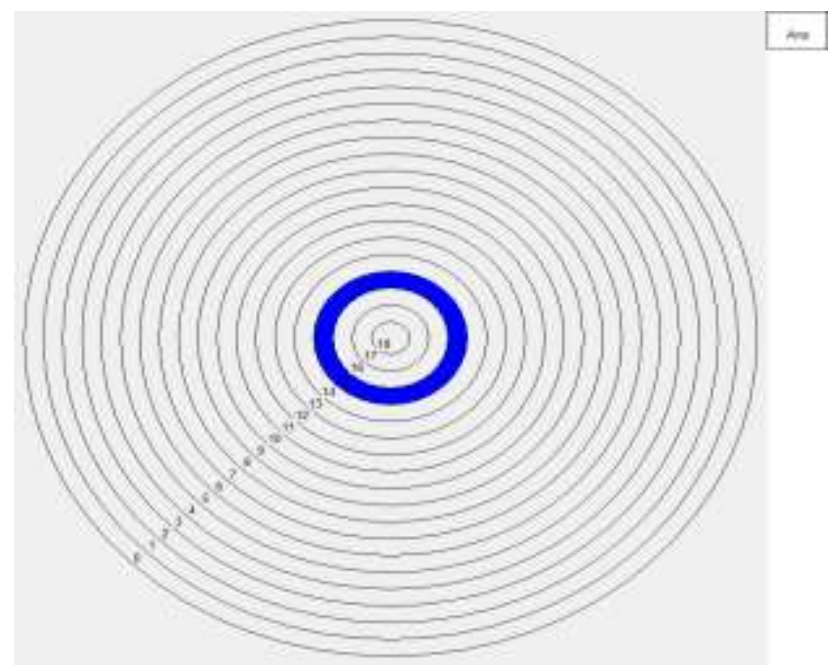

Gráfico 3. Elecciones negativas.

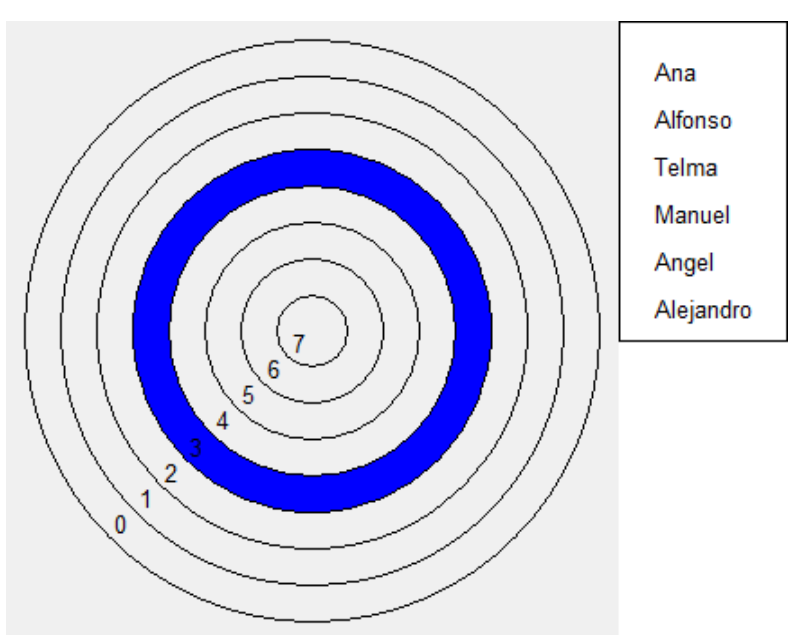

Gráfico 2. Elecciones positivas

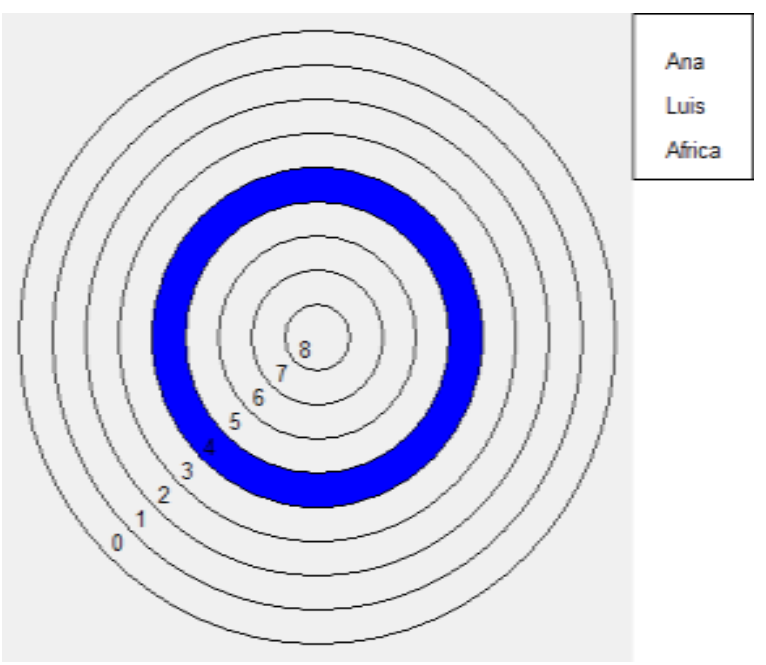

Gráfico 4. Elecciones negativas

Del mismo modo, a nivel grupal hay una mejoria en las elecciones, pues los alumnos se han elegido entre ellos de forma homogénea.

En lo referente a su preferencia para pasar el Tiempo Libre, a continuación se refleja en la tabla 6 . 
Tabla 6.

Resultado sociograma Tiempo Libre

\begin{tabular}{lll}
\hline & Antes & Después \\
\hline $\begin{array}{l}\text { Elecciones } \\
\text { positivas }\end{array}$ & $\begin{array}{l}\text { Se observa en el Gráfico 5 } \\
\text { cómo ningún compañero elige } \\
\text { a Ana. Observándose un } \\
\text { rechazo social por parte de } \\
\text { sus compañeros. }\end{array}$ & $\begin{array}{l}\text { Mientras que en segundo } \\
\text { sociograma, Gráfico 6, 3 } \\
\text { alumnos han elegido a Ana para } \\
\text { pasar el tiempo libre. }\end{array}$ \\
$\begin{array}{l}\text { Elecciones } \\
\text { negativas }\end{array}$ & $\begin{array}{l}\text { En el primer sociograma, 8 } \\
\text { compañeros la eligen de forma } \\
\text { negativa, para no pasar el } \\
\text { tiempo libre con ella, Gráfico }\end{array}$ & $\begin{array}{l}\text { En el segundo sociograma, } \\
\text { Gráfico 8, Ana es seleccionada 5 } \\
\text { veces, menor en comparación } \\
\text { con el primer sociograma. }\end{array}$ \\
& \begin{tabular}{l} 
7. \\
\hline
\end{tabular}
\end{tabular}

A continuación se reflejan los resultados en forma de gráficos, donde aparecen las elecciones positivas y negativas antes y después de la intervención.

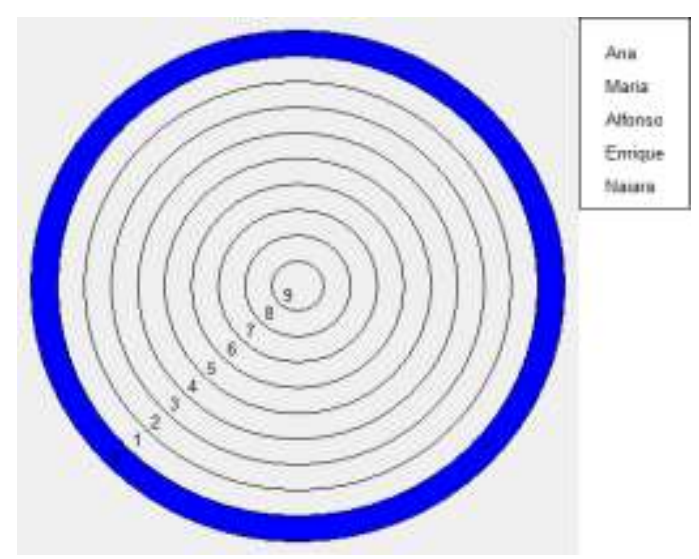

Gráfico 5. Elecciones positivas

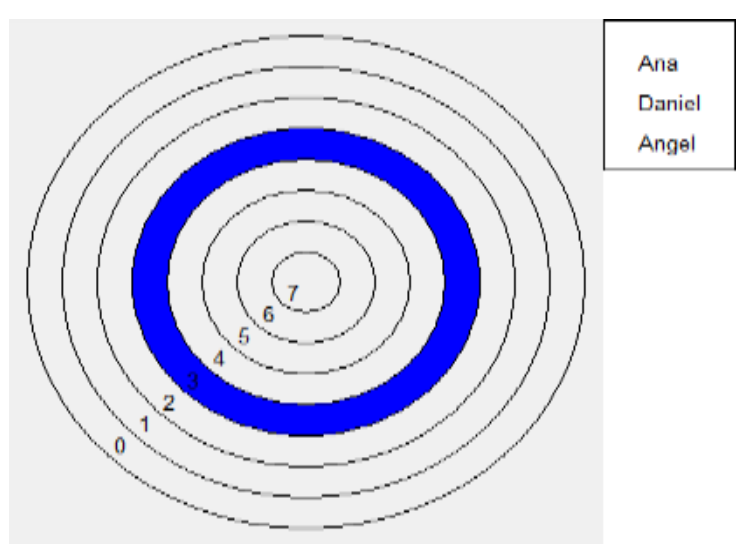

Gráfico 6. Elecciones positivas 


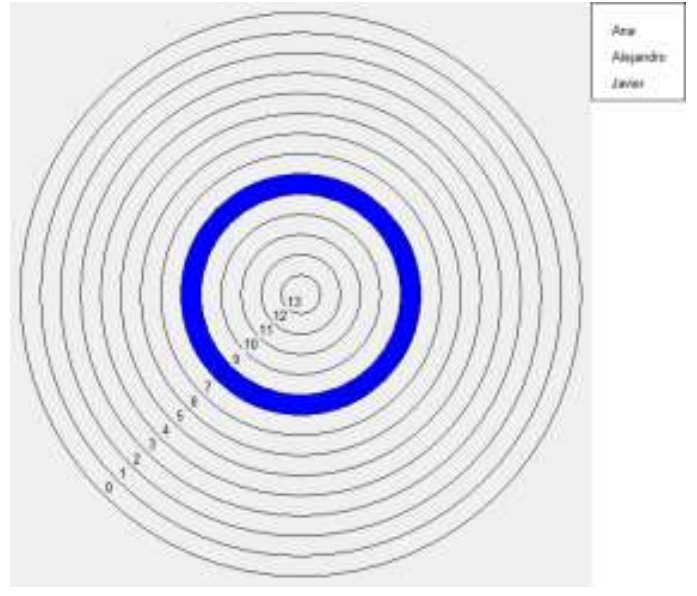

Gráfico 7. Elecciones negativas

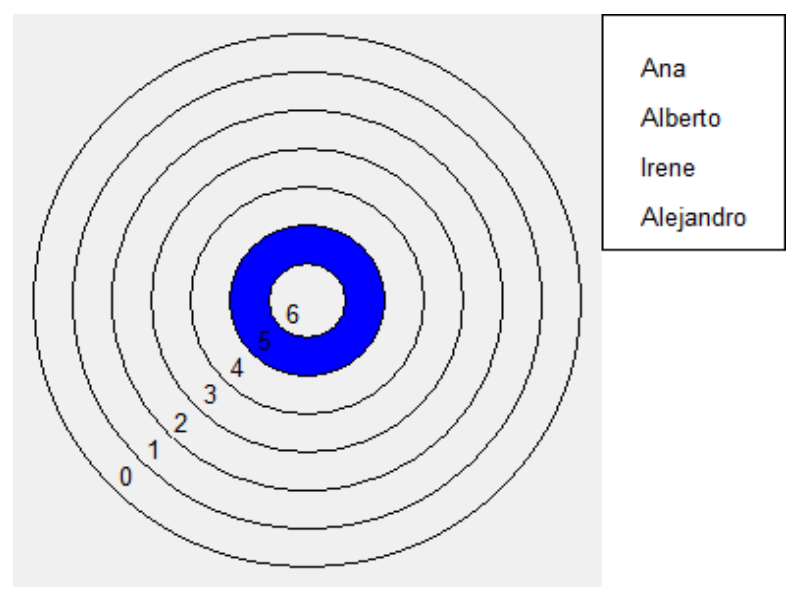

Gráfico 8. Elecciones negativas

En lo referente a sus preferencias para Sentarse en Clase, a continuación se reflejan los datos obtenidos antes y después de la intervención en la tabla 7 .

Tabla 7.

Resultado Sentar en Clase

\begin{tabular}{|c|c|c|}
\hline & Antes & Después \\
\hline $\begin{array}{l}\text { Elecciones } \\
\text { positivas }\end{array}$ & $\begin{array}{l}\text { En el primer sociograma, } \\
\text { Gráfico 9, ningún } \\
\text { compañero la elige para } \\
\text { sentarse en clase. }\end{array}$ & $\begin{array}{l}\text { Tras la intervención, se puede ver } \\
\text { en el Gráfico } 10 \text { elecciones } \\
\text { positivas, como Ana es elegida por } 2 \\
\text { compañeros para sentarse en clase. }\end{array}$ \\
\hline $\begin{array}{l}\text { Elecciones } \\
\text { negativas }\end{array}$ & $\begin{array}{l}\text { Ana es la alumna a la que } \\
\text { más compañeros han } \\
\text { elegido para no sentarse, } \\
\text { Gráfico } 11 . \text { No la quieren } 14 \\
\text { compañeros }\end{array}$ & $\begin{array}{l}\text { En cuanto al segundo sociograma, } \\
\text { Gráfico } 12 \text { de elecciones negativas, } \\
\text { Ana fue elegida } 4 \text { veces. }\end{array}$ \\
\hline
\end{tabular}


Estos resultados se reflejan en forma de gráficos a continuación:

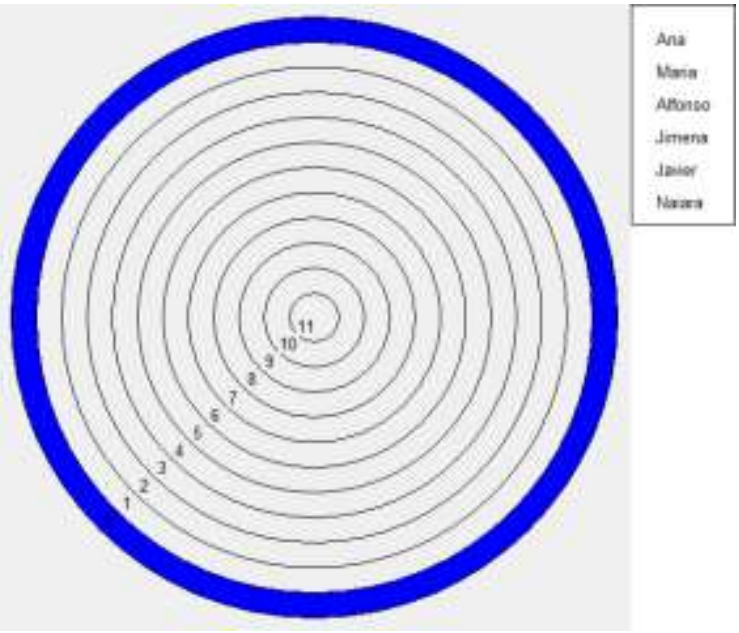

Gráfico 9. Elecciones positivas

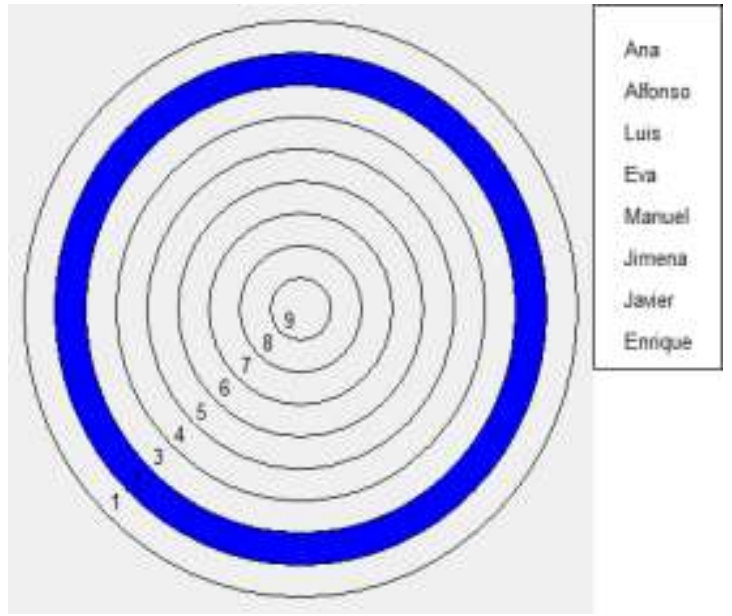

Gráfico 10. Elecciones positivas

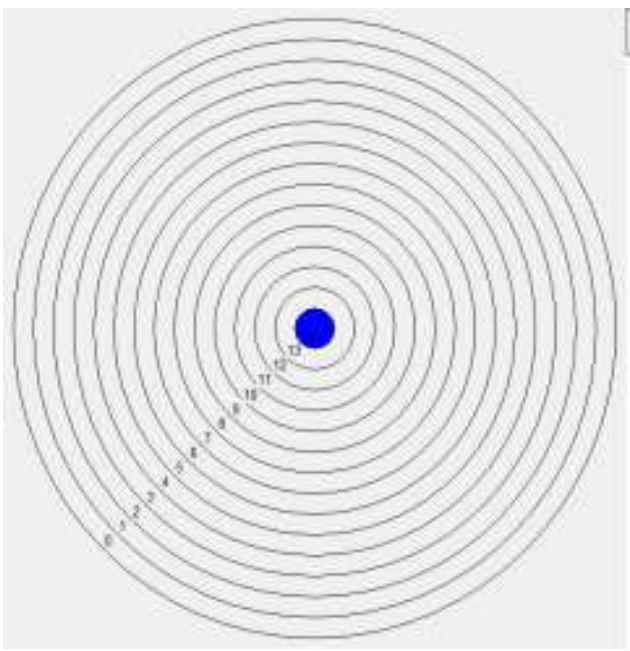

Gráfico 11. Elecciones negativas
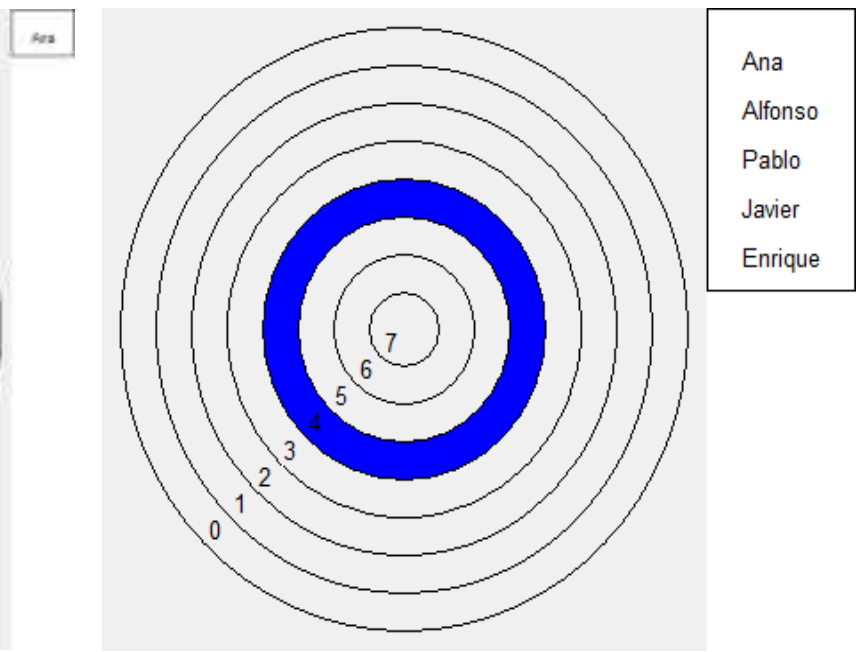

Gráfico 12. Elecciones negativas

\section{Alumna 2}

En lo referente a las preferencias para Trabajar en Grupo, se presenta en forma de resumen en la tabla 8. 
Tabla 8.

Resultado sociograma Trabajar en Grupo

\begin{tabular}{lll}
\hline & Antes & Después \\
\hline Elecciones & Se observa en la Gráfico 13, & En el segundo sociograma, Gráfico \\
positivas & como a Coral solo la elige 1 & 14,6 alumnos han elegido a Coral \\
& compañero & para Trabajar en Grupo. \\
Elecciones & En el primer sociograma, & En el segundo sociograma, Gráfico \\
negativas & Gráfico 15, se observa cómo & 16,2 alumnos no quieren a Coral \\
& $\begin{array}{l}\text { 9 compañeros la eligen para } \\
\text { no trabajar con ella. }\end{array}$ & \\
\hline
\end{tabular}

Estos resultados se reflejan a continuación en forma de gráficos, donde aparecen las elecciones positivas y negativas antes y después de la intervención.

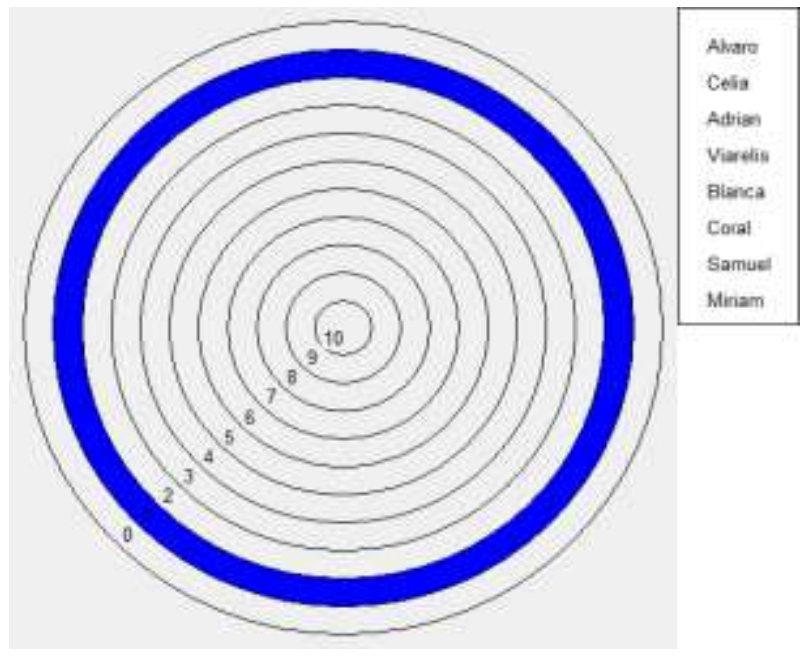

Gráfico 13. Elecciones positivas

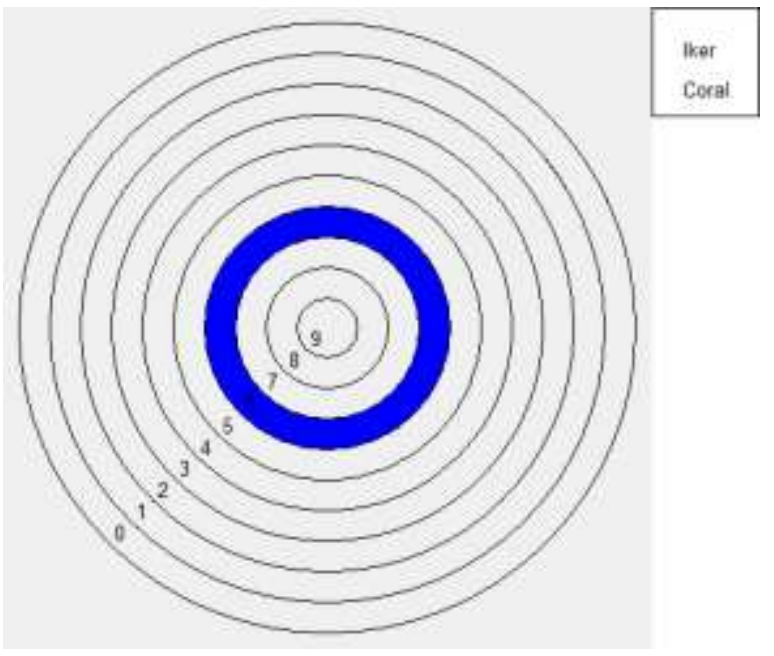

Gráfico 14. Elecciones positivas 


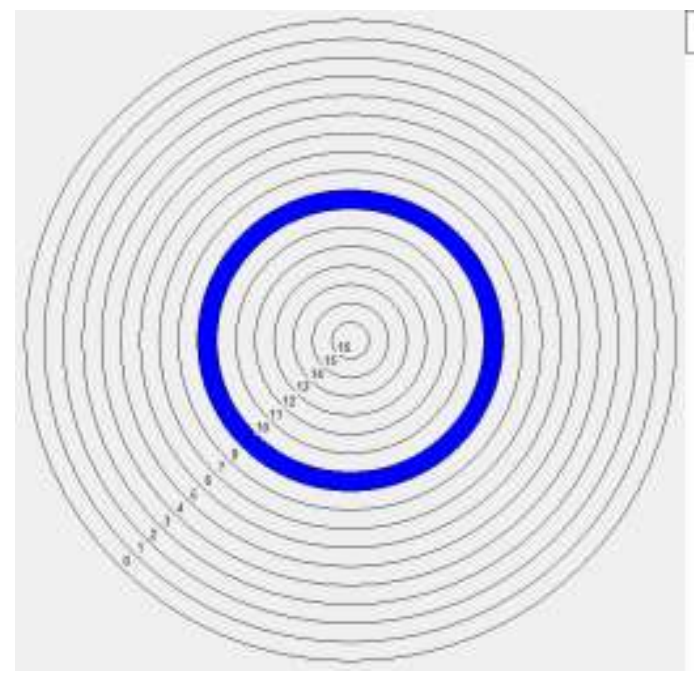

Gráfico 15. Elecciones negativas
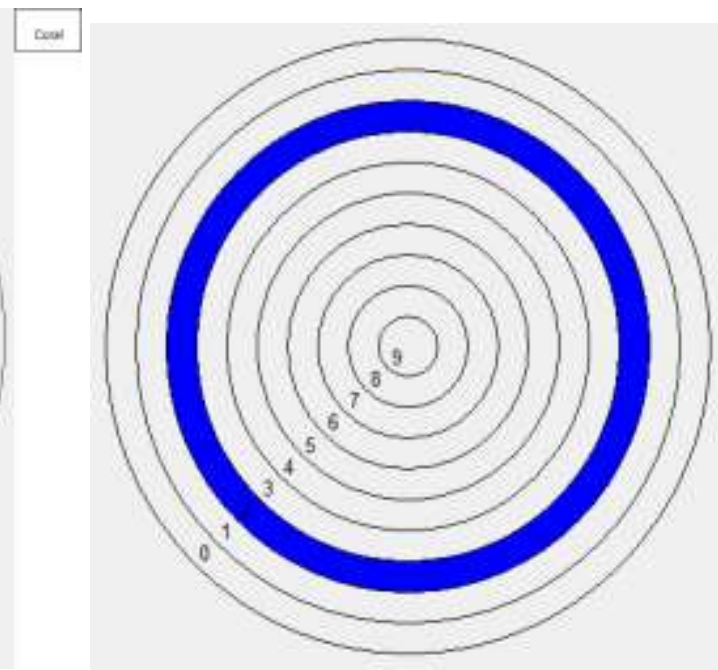

Gráfico 16. Elecciones negativas

En cuanto a la preferencia para pasar el Tiempo Libre se reflejan los datos obtenidos antes y después de la intervención en la tabla 9.

\section{Tabla 9}

Resultado sociograma Tiempo Libre

\begin{tabular}{lll}
\hline Alecciones & El Gráfico 17 refleja que & Después \\
positivas & $\begin{array}{l}\text { Tras la intervención, Gráfico 18, se } \\
\text { 2 compañeros. }\end{array}$ & $\begin{array}{l}\text { observa cómo 5 compañeros la han } \\
\text { elegido como una de las preferidas } \\
\text { para pasar el tiempo libre. }\end{array}$ \\
$\begin{array}{l}\text { Elecciones } \\
\text { negativas }\end{array}$ & $\begin{array}{l}\text { En el Gráfico 19, Coral } \\
\text { tiene hasta 8 elecciones } \\
\text { negativas. }\end{array}$ & $\begin{array}{l}\text { Mientras que en el segundo } \\
\text { sociograma, el Gráfico 20, Coral ha } \\
\text { sido elegida solo 2 veces. }\end{array}$ \\
\hline
\end{tabular}


A continuación se reflejan estos datos en los gráficos.

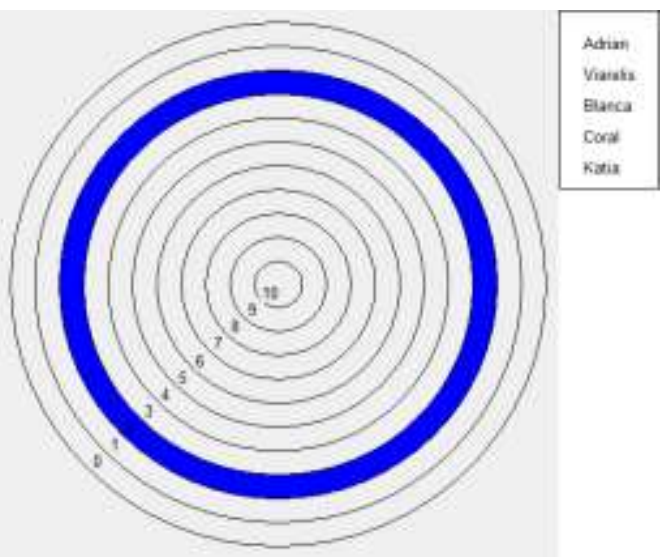

Gráfico 17. Elecciones positivas

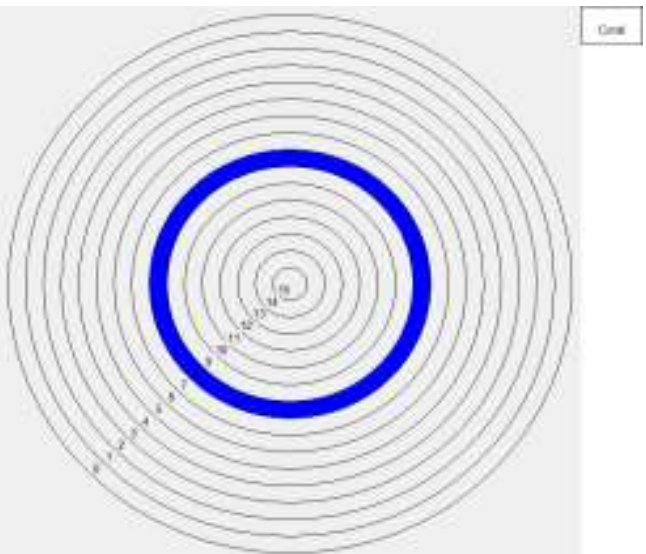

Gráfico 19. Elecciones negativas

En lo referente a su preferencia para Sentar en Clase, los datos se muestran de forma comparativa en la tabla 10.

Tabla 10

Resultado Sentar en Clase

\begin{tabular}{|c|c|c|}
\hline & Antes & Después \\
\hline Elecciones & El Gráfico 21 refleja que & Mientras que en el Gráfico 22 \\
\hline positivas & $\begin{array}{l}\text { Coral ha sido elegida por } 2 \\
\text { de sus compañeros. }\end{array}$ & $\begin{array}{l}\text { aparece reflejado cómo } 5 \text { alumnos } \\
\text { quieren sentarse con ella. }\end{array}$ \\
\hline Elecciones & El primer sociograma, & En el segundo sociograma, Gráfico \\
\hline negativas & Gráfico 23, muestra que & 24, Coral solo ha sido elegida 2 \\
\hline & $\begin{array}{l}\text { Coral ha sido elegida hasta } \\
9 \text { veces. }\end{array}$ & veces. \\
\hline
\end{tabular}


Estos resultados aparecen reflejados en los siguientes gráficos.

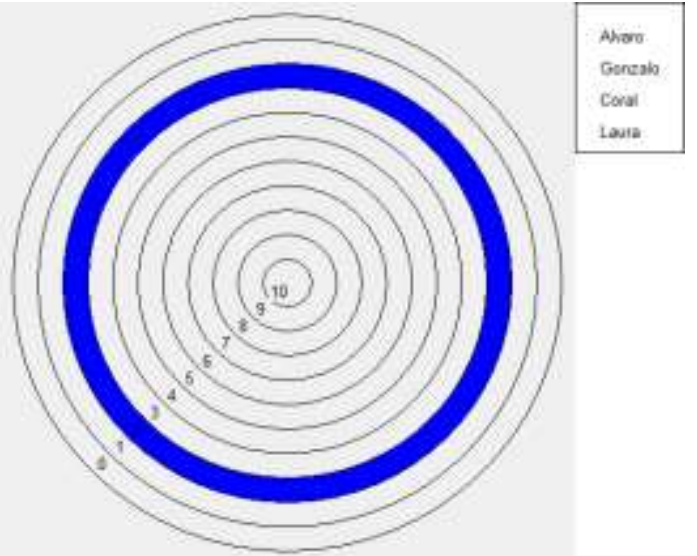

Gráfico 21. Elecciones positivas

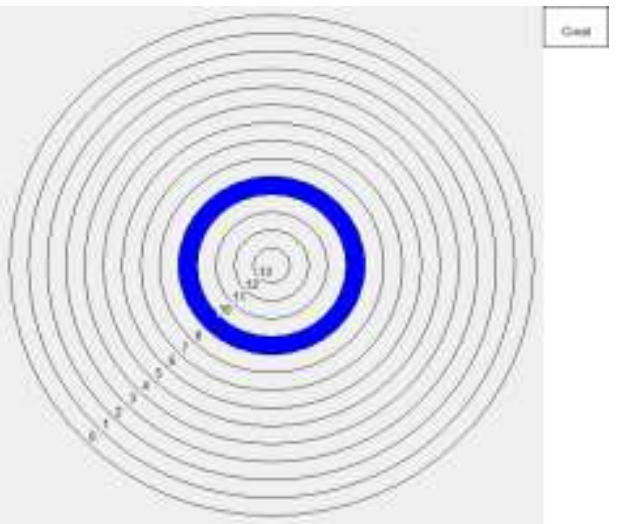

Gráfico 23. Elecciones negativas

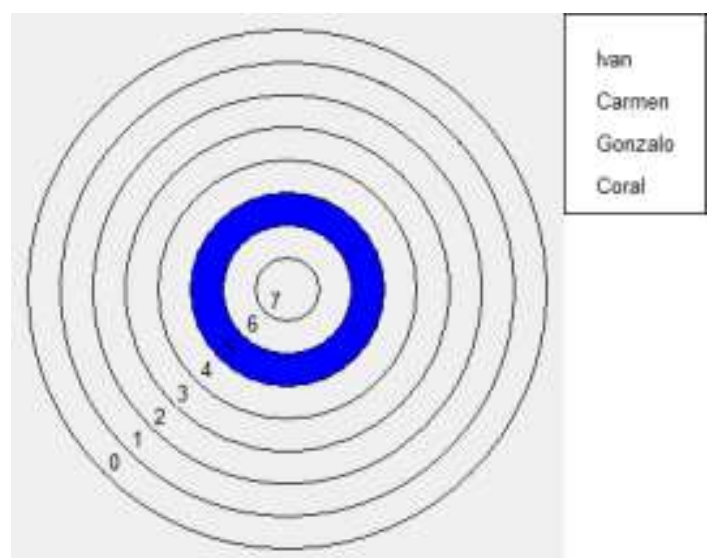

Gráfico 22. Elecciones positivas

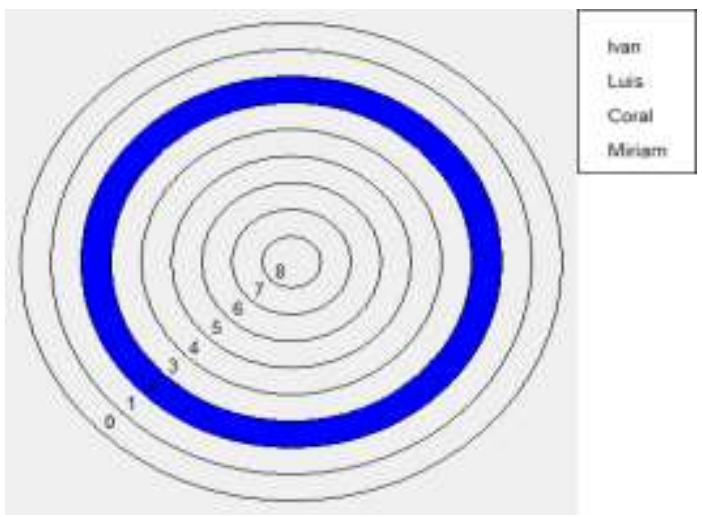

Gráfico 24. Elecciones negativas

A nivel grupal las elecciones son homogéneas, apenas hay dos alumnos que resaltan de forma negativa sobre el resto, esto refleja mejoría.

Del mismo modo, los Cuestionarios de Habilidades sociales, permitieron corroborar determinados beneficios sociales en ambos casos.

Para ello se emplearon tres cuestionarios diferentes que nos permitieron evaluar la conducta socialmente hábil y la asertividad.

Algunos de ellos fueron cumplimentados por las propias alumnas, sin embargo, debido a la baja edad de los dos casos, otros tuvieron que ser cumplimentados por padres y tutores.

Uno de ellos es Recursos para la atención a la diversidad.

Este cuestionario permitió obtener porcentajes de conducta socialmente 
hábil, incluyendo variables relacionadas con sus propios derechos y las pautas sociales de interacción. A continuación se reflejan de forma resumida en las tablas 11 y 12 .

\section{Alumna 1}

Tabla 11.

Resultados Recursos para la atención a la diversidad antes

\begin{tabular}{cccc}
\hline Padre & Madre & Tutora & Ana \\
\hline $40 \%$ & $37,5 \%$ & $32,5 \%$ & $35 \%$ \\
\hline
\end{tabular}

Tabla 12.

Resultados Recursos para la atención a la después

Los porcentajes de sociabilidad son realmente bajos antes de la intervención, tras ella se obtuvieron ciertas mejoras.

\section{Alumna 2}

Las tabla 13 refleja cómo los porcentajes obtenidos antes de la intervención son bajos; sin embargo, tras la intervención, tabla 14, sus valores aumentaron, reflejando un aumento de conducta social.

Tabla 13.

Resultados Recursos para la atención a la diversidad antes

\begin{tabular}{llll}
\hline Padre & Madre & Tutora & Coral \\
\hline $32,5 \%$ & $35 \%$ & $20 \%$ & $32,5 \%$ \\
\hline
\end{tabular}

Tabla 14.

Resultados Recursos para la atención a la diversidad después

\begin{tabular}{llll}
\hline Padre & Madre & Tutora & Coral \\
\hline $65 \%$ & $70 \%$ & $62,5 \%$ & $72,5 \%$ \\
\hline
\end{tabular}

Otro de ellos fue el Cuestionario Goldstein. Debido a la baja edad de los dos casos, este cuestionario fue cumplimentado por padres y tutores. Con esta escala se pretendía evaluar habilidades sociales generales, incluyendo la conducta interpersonal y la capacidad de resolución de conflictos.

\section{Alumna 1}

Los porcentajes obtenidos en la tabla 15 reflejaron una deficiencia en sus habilidades sociales en todos los grupos. Tras la intervención, se vieron mejoras considerables en la tabla 16. 
Tabla 15

Resultados Cuestionario Goldtein

\begin{tabular}{lllllll}
\hline Antes & Grupo I & Grupo II & $\begin{array}{l}\text { Grupo } \\
\text { III }\end{array}$ & $\begin{array}{l}\text { Grupo } \\
\text { IV }\end{array}$ & $\begin{array}{l}\text { Grupo } \\
\text { IV }\end{array}$ & $\begin{array}{l}\text { Grupo } \\
\text { VI }\end{array}$ \\
\hline Padre & $37,5 \%$ & $36,6 \%$ & $31,4 \%$ & $35,5 \%$ & $31,5 \%$ & $30 \%$ \\
Madre & $45 \%$ & $40 \%$ & $37,1 \%$ & $37,7 \%$ & $28,3 \%$ & $27,5 \%$ \\
Tutora & $42,5 \%$ & $33,3 \%$ & $34,2 \%$ & $37,7 \%$ & $30 \%$ & $27,5 \%$ \\
\hline
\end{tabular}

Tabla 16.

Resultados Cuestionario Goldtein

\begin{tabular}{lllllll}
\hline Después & Grupo I & Grupo II & $\begin{array}{l}\text { Grupo } \\
\text { III }\end{array}$ & $\begin{array}{l}\text { Grupo } \\
\text { IV }\end{array}$ & $\begin{array}{l}\text { Grupo } \\
\text { IV }\end{array}$ & $\begin{array}{l}\text { Grupo } \\
\text { VI }\end{array}$ \\
\hline Padre & $72,5 \%$ & $80 \%$ & $71,4 \%$ & $75,5 \%$ & $65 \%$ & $62,5 \%$ \\
Madre & $70 \%$ & $76,6 \%$ & $74,2 \%$ & $77,7 \%$ & $58,3 \%$ & $60 \%$ \\
Tutora & $77,5 \%$ & $80 \%$ & $80 \%$ & $80 \%$ & $66,6 \%$ & $62,5 \%$ \\
\hline
\end{tabular}

Las puntuaciones directas iniciales reflejaron baja habilidad social, con puntos: padre (77), madre (78) y tutora (76). Las puntuaciones posteriores reflejaron buena habilidad social: padre (167), madre (173) y tutora (175).

\section{Alumna 2.}

Los porcentajes obtenidos inicialmente en la tabla 17 reflejaron una baja habilidad social; mientras que tras la intervención las mejoras fueron considerables en todos los grupos, como se refleja en la tabla 18.

Tabla 17.

Resultados Cuestionario Goldtein

\begin{tabular}{lllllll}
\hline Antes & Grupo I & Grupo II & Grupo III & Grupo IV & Grupo IV & Grupo VI \\
\hline Padre & $37,5 \%$ & $40 \%$ & $34,2 \%$ & $33,3 \%$ & $31,6 \%$ & $37,5 \%$ \\
Madre & $37,5 \%$ & $46,6 \%$ & $31,4 \%$ & $31,1 \%$ & $28,3 \%$ & $35 \%$ \\
Tutor & $40 \%$ & $36,6 \%$ & $34,2 \%$ & $35,5 \%$ & $30 \%$ & $40 \%$ \\
\hline
\end{tabular}

Tabla 18.

Resultados Cuestionario Goldtein

\begin{tabular}{lllllll}
\hline Después & Grupo I & Grupo II & Grupo III & Grupo IV & Grupo IV & Grupo VI \\
\hline Padre & $72,5 \%$ & $80 \%$ & $71,4 \%$ & $71,1 \%$ & $61,6 \%$ & $75 \%$ \\
Madre & $80 \%$ & $80 \%$ & $80 \%$ & $73,3 \%$ & $63,3 \%$ & $67,5 \%$ \\
Tutora & $72,5 \%$ & $80 \%$ & $74,2 \%$ & $77,7 \%$ & $65 \%$ & $77,5 \%$ \\
\hline
\end{tabular}

Las puntuaciones directas iniciales reflejaron bajo nivel social con porcentajes del padre (76), madre (77) y tutor (70). Mientras que tras la intervención se vio buena conducta social: padre (168), madre (178) y tutor (176). 
El último, fue el cuestionario CABS o Escala de comportamiento asertivo para niños, que permitió evaluar la conducta asertiva en contraposición de otras conductas socialmente inadecuadas como la inhibición o agresividad.

\section{Alumna 1.}

La tabla 19 refleja cómo la alumna comenzó con un perfil de inhibición, seguido de valores agresivos. Tras la intervención hubo una conducta más asertiva, disminuyendo el perfil inhibitorio.

Tabla 19.

Resultados CABS

Antes

Valores agresividad 7 puntos de 24

Valores asertividad 6 puntos de 24

Valores inhibición 11 puntos de 24

El perfil inicial reflejó inhibición conductual.
Después

Valores agresividad 2 puntos de 24

Valores asertividad 15 puntos de 24

Valores inhibición 7 puntos de 24

La asertividad aumentó tras la intervención.

\section{Alumna 2.}

En este caso, la tabla 20 reflejaba antes de la intervención un perfil inhibitorio, tras la intervención, aumentó considerablemente la conducta asertiva.

Tabla 20.

Resultados CABS

Antes

Valores agresividad 5 puntos de 24

Valores asertividad 6 puntos de 24

Valores inhibición 13 puntos de 24

El perfil inicial reflejó inhibición conductual.
Después

Valores agresividad 3 puntos de 24

Valores asertividad 12 puntos de 24

Valores inhibición 8 puntos de 24 Hubo una disminución de su inhibición y un aumento de la asertividad.

¿Es necesario intervenir en ciertos aspectos conductuales relacionados con el TDAH para conseguir beneficios sociales?

Las entrevistas con padres y profesores, así como la observación directa reflejaron cómo hubo progresos a nivel conductual. Y cómo su mejora influía en sus mejoras sociales.

Estos datos fueron analizados con Nudist y se presentan de forma abreviada en tabla sus indicadores. 
Además, la tabla 21, permite complementar la cuestión tratada con anterioridad.

Tabla 21.

Resultados Nudist

\begin{tabular}{ll}
\hline Progresos & No hubo progresos \\
\hline Comunicación en grupo & Liderazgo \\
Comunicación con adultos & Mirar a la cara cuando se comunica \\
Retraimiento & \\
Problemas de integración & \\
Escuchar cuando se la habla & \\
Reducida red de relaciones sociales & \\
Problemas de relación con sus iguales & \\
Problemas de relación con los adultos & \\
Ignorar & \\
Aceptar críticas & \\
Resolución de conflictos & \\
Timidez & \\
Interés social & \\
Empatía &
\end{tabular}

Además, se vieron mejoras en la conducta de ambos casos, como se refleja la tabla 22. Mejoras que pudieron influir en los aspectos sociales.

Tabla 22.

Resultados Nudist

\begin{tabular}{ll}
\hline Progresos & No hubo progresos \\
\hline Conducta disruptiva & Rabietas \\
Cumplimiento de las normas & \\
Cambios del estado de ánimo & \\
Frustración & \\
Problemas de autocontrol & \\
Autocontrol de las emociones & \\
\hline
\end{tabular}

¿Se puede llevar a cabo una intervención en el ámbito escolar sin alterar la dinámica académica?

Los datos obtenidos reflejan mejoras sociales y conductuales tras intervenir en el ámbito escolar. Todo esto permite corroborar la pregunta hipotética de partida, donde se reflejaba que es posible intervenir sin alterar la dinámica académica. La intervención planteada puede ser llevada a cabo por los propios tutores si se sigue el procedimiento llevado a cabo. 


\section{Conclusiones y discusión}

Muchos de los niños con TDAH tienen dificultades sociales y baja competencia social, en muchas ocasiones son rechazados por sus conductas inadecuadas (Bishop, Mulraney, Rinehart y Sciberras, 2019).

Es frecuente que estos problemas estén presentes en los primeros años de vida y se agraven con la edad (Willis, Siceloff, Morse, Neger y Flory, 2019, y Fox, Dishman, Valicek, Ratcliff y Hilton, 2020).

Como vimos en la introducción, los fármacos son insuficientes para mejorar las relaciones interpersonales, como lo demuestra la literatura (Jarque-Fernández, 2012 o Aduen, Day, Kofler, Harmon, Wells y Sarver, 2018).

Los primeros resultados obtenidos referidos a las mejoras sociales en estos dos casos, parecen confirmar las hipótesis de partida, algunos niños con TDAH tienen dificultades sociales y es necesaria su intervención (Colomer, 2016, Storebo, et al., 2019 y Fox, et al., 2020). Como vemos en los sociogramas, en las escalas y en Nudist, se encontraron mejoras tanto en las habilidades sociales como en las conductas en ambos casos. Todas estas mejoras, así como las técnicas aplicadas directamente por profesores y padres contribuian a mejorar determinados síntomas del TDAH.

Los indicadores que expresaron una clara mejora y resultados favorables fueron (habilidades sociales: nivel de comunicación, interés social, integración, red social, resolución de conflictos, interacción con iguales $y$ adultos, y empatia). Sin obtener cambios en variables como liderazgo o mirar a la cara cuando se establece una comunicación. En lo referente a la categoria conducta desadaptativa, apenas hubo cambios en rabietas, pues es una manera de captar la atención, sin embargo, se encontraron resultados favorables en conducta adaptativa en el cumplimiento de las normas, en frustración y en autocontrol (emocional y conductual).

Con estos resultados se pudo dar respuesta a algunas cuestiones planteadas incialmente, cómo la posibilidad de obtener beneficios sociales en el contexto escolar, relacionándolo así con estudios previos (Russo, Bakker, Rubiales y Betina-Lacunza, 2019 o Fox, et al., 2020) o la necesidad de intervenir en aspectos conductuales que pudieran influir en la sociabilidad, como hemos añadido desde nuestro estudio.

Estos resultados son congruentes con los presentados en otras investigaciones, donde se manifiesta la necesidad de reforzar el área social y en concreto las habilidades sociales de este alumnado a través de programas específicos de intervención (Jarque-Fernández, 2012; Aduen, et al., 2018; Kofler, Harmon, Aduen, Day, Austin, Spiegel, Irwin y Sarver, 2018; Bishop, et al., 2019, o Fox, et al., 2020).

Partiendo de esta premisa y como vemos en los resultados, es necesario intervenir no solo en las habilidades sociales, sino en los aspectos conductuales de estos sujetos, ya que pueden influir 
directamente en su sociabilidad (Lora y Moreno, 2008; Ronk, Hund y Landau, 2011; Bishop, et al., 2019 y Fox, et al., 2020).

Es así, cómo se ha querido demostrar no solo las dificultades sociales de estos dos casos con TDAH, sino también los aspectos positivos y las mejoras tras la intervención de un programa elaborado para que sean los propios tutores los que puedan aplicarlo sin necesidad de un profesional externo.

Cuando vimos los tratamientos dentro de la introducción, el más efectivo y utilizado es el combinado, donde se da importancia a los aspectos psicológicos, sociales y conductuales. Los resultados obtenidos en este trabajo coinciden en ciertos aspectos con este hecho, cuando se ven las mejoras sociales y conductuales obtenidas, añadiendo la necesidad de intervenir en ambas. No sólo por ser aspectos fundamentales en la vida, sino porque de forma indirecta se influye en algunos aspectos del TDAH, como así se reflejan en los resultados obtenidos en las entrevistas y observación.

Estudios como el de Colomer (2016); el de Liesa-Orús, LatorreCosculluela y Vázquez-Toledo (2017); Aduen, et al., (2018), o el de Bishop, et al., (2019), reflejan la necesidad e importancia de intervenir en las habilidades sociales. Sin embargo, no hay que tener en cuenta solo este concepto para mejorar en sociabilidad, sino que, como se ha visto en los resultados, también es necesario intervenir en la conducta pues ambos aspectos están relacionados.

Con este estudio se pretende aportar nuevos datos sobre las mejoras de niños con TDAH en el ámbito académico a través de una intervención psicosocial. El interés de dicha investigación reside en que ofrece otra manera de que los sujetos con TDAH mejoren sus habilidades sociales.

A lo largo del estudio se encontraron con limitaciones que podemos resumir así. Por un lado se ampliaría el foco del objeto de estudio si ampliáramos la muestra, es decir, el número de alumnado en su aplicación, aún no siendo ese el objetivo de dicho trabajo, podría aportar más matices acerca de cómo implementar este tratamiento con alumnado con TDAH.

Derivado de estas limitaciones aportamos nuevas líneas de investigación que se derivan de tales déficits encontrados: (a) Realizar un estudio estadístico en diferentes centros en los que se aplique dicha intervención. (b) Orientar la intervención hacia aspectos emocionales en estos casos. (c) Introducir a la intervención más técnicas específicas acordes a la sintomatología TDAH.

En nuestro país son escasas las intervenciones sobre este tema en el ámbito académico, a pesar del elevado número de casos. Los niños con TDAH reciben medicación y en el mejor de los casos los padres pueden permitirse una intervención psicopedagógica, dejando de lado el lugar donde más horas al día pasan y donde más dificultades muestran, el colegio. 
Por todo ello, con este trabajo se pretende contribuir a aumentar el número de investigaciones en nuestro país, aportando datos a la investigación científica y al conocimiento del TDAH.

Con todo esto, podriamos considerar que esta investigación puede ser relevante no solo para las familias y sujetos que presentan TDAH, sino también para los profesionales del ámbito escolar, ya que se ofrecen datos contrastados de los beneficios que este tipo de intervenciones ofrecen.

\section{Referencias}

Aduen, P. A., Day, T. N., Kofler, M. J., Harmon, S. L., Wells, E. L., y Sarver, D. R. (2018). Social problems in ADHD: Is it a skills acquisition or performance problem? Journal Psychopathology and Behavioral Assessment, 40, 440-451. doi: https://doi.org/10.1007/s10862-018-9649-7

American Psychiatric Association. (2013). Diagnostic and statistical manual of mental disorders (5a ed.). Barcelona: Masson.

Barkley, R. A. (2011). Niños hiperactivos: Cómo comprender y atender sus necesidades especiales. Barcelona: Paidós Ibérica.

Becker, S. P., Garner, A. A., Tamm, L., Antonini, T. N., y Epstein, J. N. (2019). Honing in on the social difficulties associated with sluggish cognitive tempo in children: Withdrawal, peer ignoring, and low engagement. Journal of Clinical Child \& Adolescent Psychology, 48(2), 228-237.

Bishop, C., Mulraney, M., Rinehart, N., y Sciberras, E. (2019). An examination of the association between anxiety and social functioning in youth with ADHD: A systematic review. ScienceDirect, 273, 402-421.

doi: https://doi.org/10.1016/j.psychres.2019.01.039

Colomer, C., Miranda, A., Berenguer, C., y Palomero, B. (2016). Funcionamiento social en niños con TEA y TDAH. Psicología y Educación. Presente y Futuro, 665-672. Alicante: ACIPEAsociación Científica de Psicología y Educación.

Creswell, J. W. (2014). Research design: Qualitative, quantitative and mixed methods approaches. Thousand Oaks, 12(5), 1-342. 
Fox, A., Dishman, S., Valicek, M., Ratcliff, K., y Hilton, C. (2020). Effectiveness of social skills interventions incorporating peer interactions for children with attention deficit hyperactivity disorder: A systematic review. American Journal of Occupational Therapy, 74(2). doi: https://doi.org/10.5014/ajot.2020.040212

Garrido-Landivar, E. (2017). 500 preguntas y respuestas sobre la Hiperactividad (TDAH). Pamplona: Ediciones Eunate.

Jarque-Fernández, S. (2012). Eficacia de las intervenciones con niños $\mathrm{y}$ adolescentes con trastorno por déficit de atención con hiperactividad (TDAH). Anuario de Psicología, 42(1), 19-33.

Kofler, M. J., Harmon, S. L., Aduen, P. A., Day, T. N., Austin, K. E., Spiegel, J. A., ... Sarver, D. E. (2018). Neurocognitive and behavioral predictors of social problems in ADHD: A Bayesian framework. Neuropsychology, 32(3), 344-355.

doi: https://doi.org/10.1037/neu0000416

Liesa-Orús, M., Latorre-Cosculluela, C., y Vázquez-Toledo, S. (2017). Habilidades sociales de niños con déficits atencionales y contextos escolares inclusivos. Revista Española de Orientación y Psicopedagogía, 28(2), 113-121.

Londoño-Paredes, D. E. (2017). El trastorno por déficit de atención con hiperactividad: Una mirada socio-cultural. Revista de la Asociación Española de Neuropsiquiatria, 37(132), 477-496. doi: https://doi.org/10.4321/S0211-57352017000200009

López, J., Montes, J., y Sánchez, A. (2003). Trastorno por Déficit de Atención con Hiperactividad: Análisis discriminante por grupos. Revista de Psiquiatría Infanto-Juvenil, 20(3), 108-119.

Lora, M. J. A. y Moreno, G. I. (2008). Perfil social de los subtipos del Trastorno por Déficit de Atención con Hiperactividad. Apuntes de Psicología, 26, 317-329.

Organización Mundial de la Salud. (1992). Clasificación estadistica Internacional de Enfermedades y Problemas Relacionados con la Salud. (Vol.2). Washington, D.C: OPS.

Parellada, M. (2009). TDAH: Trastorno por Déficit de Atención e Hiperactividad. De la infancia a la edad adulta. Madrid: Alianza Editorial. 
Puentes-Rozo, P., Jiménez-Figueroa, G., Pineda-Alhucema, W., y Montoya, D. (2014). Déficit en habilidades sociales en niños con trastorno por déficit de atención-hiperactividad, Evaluados con la Escala BASC. Revista Colombiana de Psicología, 23(1), 95-106.

Rodríguez-Salinas, E., Navas-García, M., González-Rodríguez, P., Fominaya, S., y Duelo-Marcos, M. (2006). La escuela y el trastorno por déficit de atención con/sin hiperactividad (TDAH). Revista Pediatría Atención Primaria, 8(4), 175-198.

Rojo-Amato, G, Tárraga-Mínguez, R., y Sanz-Cervera, P. (2016). La intervención en competencia social en estudiantes con TDAH: Un estudio de revisión. Revista de Investigación y Docencia Creativa, 5, 329-340.

Ronk, M., Hund, A., y Landau, S. (2011). Assessment of social competence of boys with attention deficit/hyperactivity disorder: Problematic peer entry, host responses, and evaluations. Journal of Abnormal Child Psychology, 39(6), 829-840.

Russo, D., Bakker, L., Rubiales, J., y Betina-Lacunza, A. (2019). Intervención en habilidades de solución de problemas interpersonales: Resultados preliminares en niños con diagnóstico de TDAH. Revista Chilena Neuropsicología, 14(1), 0105.

Stake, R.E. (1998). Investigación con estudio de casos ( $5^{\mathrm{a}}$ ed.). Madrid: Morata.

Storebo, O. J., Elmose-Andersen, M., Skoog, M., Joost-Hansen, S., Simonsen, E., Pedersen, N., ... Gluud, C. (2019). Social skills training for attention deficit hyperactivity disorder (ADHD) in children aged 5 to 18 years. Cochrane Database of Systematic, 6 . doi:10.1002/14651858.CD008223.pub3.

Vinuesa-Fernández, F. G. (2017). Prehistoria del TDAH: Aditivos para un diagnóstico insostenible. Papeles del Psicólogo, 38(2), 107115 .

Willis, D., Siceloff, E. R., Morse, M., Neger, E. y Flory, K. (2019). Stand-alone social skills training for youth with ADHD: A systematic review. Clinical Child and Family Psychology Review, 22(3), 348-366. doi: 10.1007/s10567-019-00291-3. 